\title{
Formation of the Layered Etch Films on AA1050 Aluminum Plates Etched in Nitric Acid Using Alternating Currents
}

\author{
C. S. Lin $^{\mathrm{a}, \mathrm{z}}$ and C. C. Chiu ${ }^{\mathrm{b}}$
}

${ }^{a}$ Department of Materials Science and Engineering, National Taiwan University, Taipei 106, Taiwan

${ }^{b}$ Taiwan Semiconductor Manufacturing Company, Hsinchu Science Park, Hsinchu 300, Taiwan

The structure of the etch film on aluminum plates etched in nitric acid was systematically studied by varying the ratio of positive to negative charges provided by a rectangular current waveform. When etched using a symmetrical square current, the plates were dotted with the etch pits inside which a layered etch film formed via the stacking of dark-contrast $v s$. light-contrast layers. The former was presumably aluminum hydroxide, while the latter was the channels that were filled with the electrolyte during etching. The thickness of the aluminum hydroxide layer increased with increasing the charge passed during the cathodic half-cycle Hydrogen discharge during the cathodic half-cycle led to the formation of the hydroxide layer. Hydrogen evolution at the interface formed microvoids and might induce defects in the hydroxide layer. Based on dissolution-passivation of the same pit, a possible mechanism was proposed to explain the formation of the layered etch film on aluminum etched in nitric acid using the alternating current with emphasis on the effects of hydrogen discharge.

(C) 2005 The Electrochemical Society. [DOI: 10.1149/1.1931448] All rights reserved.

Manuscript received August 18, 2004; revised manuscript received February 3, 2005. Available electronically June 7, 2005.

Pitting corrosion of aluminum alloys has been extensively studied in order to develop the strategies for preventing the alloys from corrosion, ${ }^{1-4}$ and to impart unique surface properties to the alloys. These surface modification techniques rely largely on the control of the formation and breakdown of the passive films on aluminum such as the production of the foils for electrolytic capacitors and the plates for lithographic printing. ${ }^{5-15}$ For example, an alternating current is generally employed to induce simultaneous film formation and incomplete destruction of the film for forming the etch pits that effectively increase the surface areas of the aluminum foils. The development of tunnel pits on aluminum etched using the dc current is also closely related to the passivation of the pit tips. ${ }^{9}$

The formation of the etch film and the passivation of existing pits on aluminum etched in hydrochloric acid using alternating currents have been demonstrated to occur during the cathodic half-cycle, whereas local pitting occurs at the weak spots on the passive film during the anodic half-cycle. ${ }^{5,7}$ General reviews ${ }^{1-3}$ of the literature related to pitting corrosion of aluminum in chloride solutions have been written and generally recognize that the pit formation involves a multistep process: adsorption of chloride ions on the oxidesolution interface, followed by dissolution of the oxide film or diffusion of chloride ions across the oxide film, and finally the dissolution of aluminum. This process might be assisted by an anodic potential, ${ }^{3}$ particularly when the potential exceeds a critical pitting potential. ${ }^{1-3,16,17}$ However, cathodic polarization might enhance the population density of the pits formed during subsequent anodic polarization above the pitting potential. ${ }^{18}$ This enhancement is attributed to the formation of aluminum hydroxide or oxyhydroxide, which has lower electric resistance for ionic conduction than the aluminum oxide. ${ }^{19}$ Moreover, hydrogen formed at the oxide-metal interface generally causes the formation and subsequent rupture of blisters in the protective film. ${ }^{1,20,21}$ As a result, anodic pitting is promoted as the protective film is perforated by the hydrogen evolution process. Apparently, hydrogen evolution during cathodic polarization either facilitates the formation of the passive film via the increase in interfacial $\mathrm{pH}$ or enhances the population density of the defects associated with the anodic oxide film or the etch film formed during the early stage of the cathodic half-cycle.

Because chloride is known as an aggressive species for inducing the pitting corrosion on most aluminum alloys, ${ }^{1-3,16}$ whereas nitrate effectively inhibits the pitting corrosion on aluminum, ${ }^{17}$ the building elements of the pits and the microstructure of the etch film differ for the aluminum etched in hydrochloric acid and nitric acid. ${ }^{12-15,22,23}$ While the pitting behavior of aluminum in chloride solutions has been extensively studied, the mechanism regarding the formation of

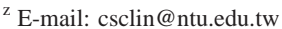

the layered etch film on the aluminum etched in nitric acid using alternating currents remains to be determined. In this study, alternating currents of square waveforms with distinct ratios of positive to negative charges were employed to investigate the formation of the etch film and its subsequent breakdown on aluminum etched in nitric acid. Both were found to be related to hydrogen discharge during the cathodic half-cycle.

\section{Experimental}

Alternating current etching. - The material used here is the 0.25-mm-thick AA1050 aluminum lithographic plate consisting of $0.28 \%$ (in weight percentage) $\mathrm{Fe}, 0.10 \% \mathrm{Si}, 0.04 \% \mathrm{Cu}, 0.005 \% \mathrm{Mn}$, $0.005 \% \mathrm{Zn}, 0.003 \% \mathrm{Ti}$, and $\mathrm{Al}$ in balance. The cross section of the plate exhibited a banded structure characteristic of the deformation along the rolling direction (Fig. 1a). Consequently, the aluminum matrix contained a high density of dislocations and subgrains with size less than $1 \mu \mathrm{m}$. Figure $1 \mathrm{~b}$ shows that the precipitates in aluminum presented as either platelets or spheres, which were marked by the arrow and double arrow in the figure, respectively. Most of the precipitates were platelets and had an average composition of $61.85 \%$ (in weight percentage) Al, 37.24\% Fe, and $0.91 \% \mathrm{Si}$ (Fig. 1c). The spherical precipitate was also composed of $\mathrm{Al}, \mathrm{Fe}$, and $\mathrm{Si}$ species and had lower Fe (19.16\%) content but higher Si (3.97\%) than the platelet one. After degreasing in $5 \mathrm{wt} \% \mathrm{NaOH}$ solution at $50^{\circ} \mathrm{C}$, the plates were etched in $0.16 \mathrm{M}$ nitric acid at $35^{\circ} \mathrm{C}$ using a flow cell. Alternating current (ac) etching was performed at a current density of $15 \mathrm{~A}_{\text {peak }} / \mathrm{dm}^{2}$ for each anodic and cathodic half-cycle at $50 \mathrm{~Hz}$, provided by a precision function generator and a TA250 power amplifier. The typical waveform used is shown in Fig. 2. In order to study the effects of the ratio of positive to negative charges on the microstructure of the etch film, $t_{\mathrm{a}} / t_{\mathrm{c}}$ was varied between 20 to $1 / 20$, where $t_{\mathrm{a}}$ and $t_{\mathrm{c}}$ are the duration time of the anodic and cathodic half-cycles, respectively. Galvanostatic etching was conducted for $57 \mathrm{~s}$ regardless of the charge ratio.

Microstructural characterization.-After etching, the surface morphology of the plates was observed using a scanning electron microscope (SEM). The detailed microstructure of the plates was characterized via cross-sectional transmission electron microscopy (TEM) specimens, which were prepared by a combination of mechanical grinding and ion-beam thinning method. The thickness of the distinct layers associated with the etch film was reported as an average of at least ten measurements made on TEM micrographs. Finally, the chemical composition and structure of the etch film were characterized using energy-dispersive spectroscopy (EDS) and the electron diffraction technique, respectively. 

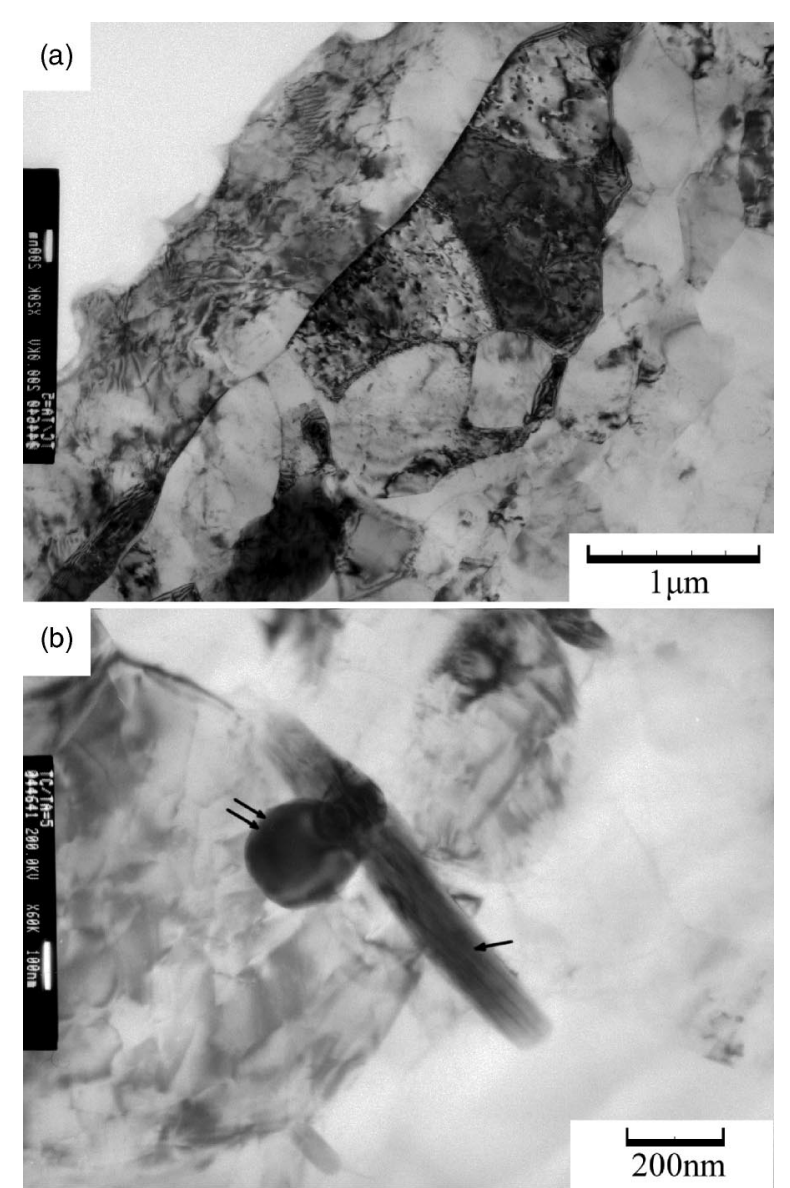

(c)

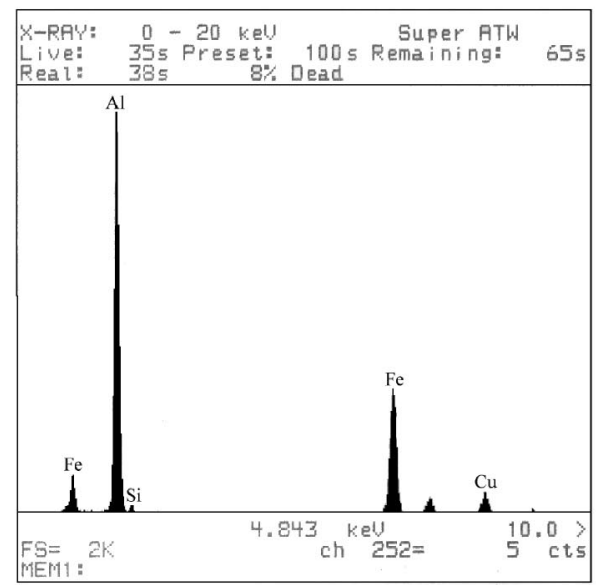

Figure 1. Cross-sectional TEM micrographs of 1050 aluminum plate showing (a) the overall grain structure, (b) the morphology of the various precipitates, and (c) EDS spectrum taken from the area marked by the arrow in (b).

\section{Results}

AA1050 plates etched using the symmetrical square current.Aluminum plates for lithographic printing are usually electrograined in hydrochloric acid or nitric acid using a proper sinusoidal alternating current. ${ }^{11-15,22,23}$ When etched using a symmetric square current, i.e., $t_{\mathrm{a}} / t_{\mathrm{c}}=1$, the aluminum plate was uniformly grained and its surface was entirely dotted with the pits (not shown here). Figure 3 shows the cross section of an etched plate, illustrating the basic building element of the etch pits was the individual hemispherical pit. This figure also shows a layered etch film covering the pit. This layered etch film apparently consisted of alternate light-contrast and dark-contrast layers. In Fig. 3 the dark-contrast layers copied the

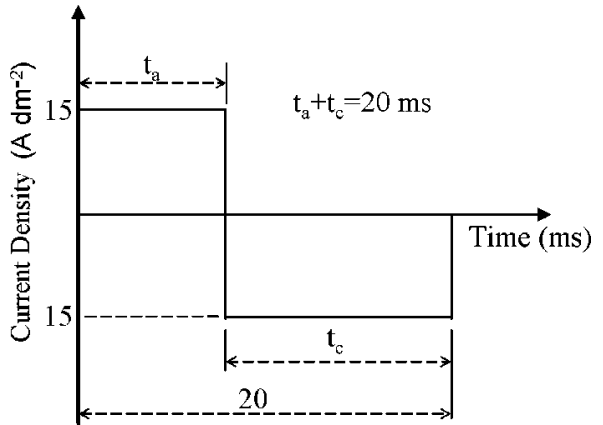

Figure 2. Schematic diagram showing the waveform of the alternating current used.

shape of the pit. Compared to the dark-contrast layer, the lightcontrast layer was porous and contained many microvoids. Since similar layered etch films have been observed on the pits formed in nitric acid via the sinusoidal current, ${ }^{13,15}$ they must be associated with aluminum etched in nitric acid regardless of the ac waveform. Like the layered etch film formed in nitric acid using the sinusoidal current, ${ }^{15}$ EDS spectrum (not shown here) taken from the darkcontrast layer consisted of aluminum and oxygen signals, suggesting the dark-contrast layer was aluminum hydroxide. Except aluminum and oxygen, the light-contrast layer mainly consisted of carbon, which is the primary constituent of the M-Bond epoxy used for sandwiching two etched plates together during sample preparation. The M-Bond epoxy apparently flowed into the light-contrast layer, signifying the light-contrast layer was porous as characterized by the TEM bright-field image (Fig. 3).

AA1050 plates etched at $t_{a} / t_{c}$ exceeding 1 .- - In order to investigate the effect of the anodic charge imposed during each anodic half-cycle on the structure of the etch film, the $t_{\mathrm{a}} / t_{\mathrm{c}}$ ratio was increased up to 20. Figures 4-6 compare the layer thickness of the etch films formed at increasing $t_{\mathrm{a}} / t_{\mathrm{c}}$. Figure 4 shows that the pits formed at $t_{\mathrm{a}} / t_{\mathrm{c}}=2$ were also covered with layered etch films. However, these etch films seemed to be fragile, particularly those residing nearby the surface (as marked by the arrow in Fig. 4a). Conversely, the layers overlaying nearby the base of the pit still assumed the shape of the pit. A close-up view of Fig. 4a shows that a darkcontrast layer intimately contacted the aluminum substrate (Fig. 4b). This layer was approximately $30 \mathrm{~nm}$ thick and was more compact than the other dark-contrast layers that did not directly contact the aluminum substrate. Moreover, this layer had peeled locally off the

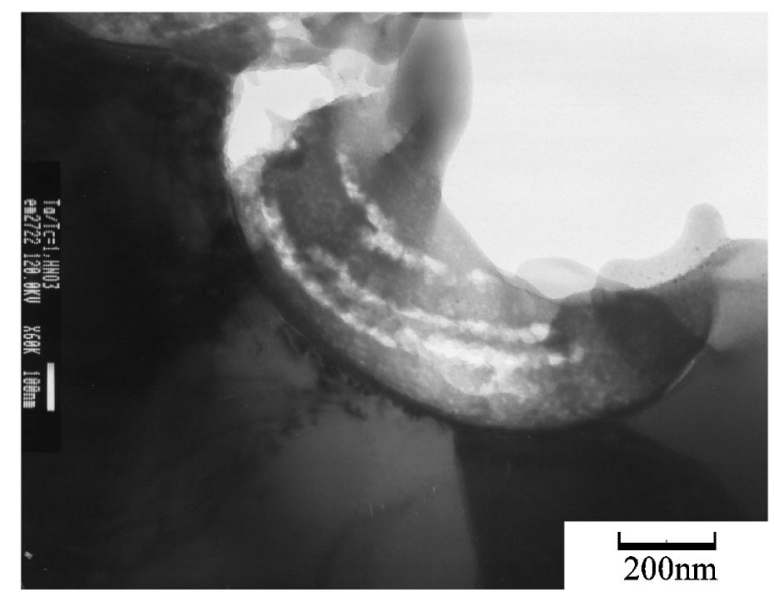

Figure 3. Cross-sectional TEM micrograph of the plate etched at $t_{\mathrm{a}} / t_{\mathrm{c}}=1$ showing the morphology of the layered etch film. 

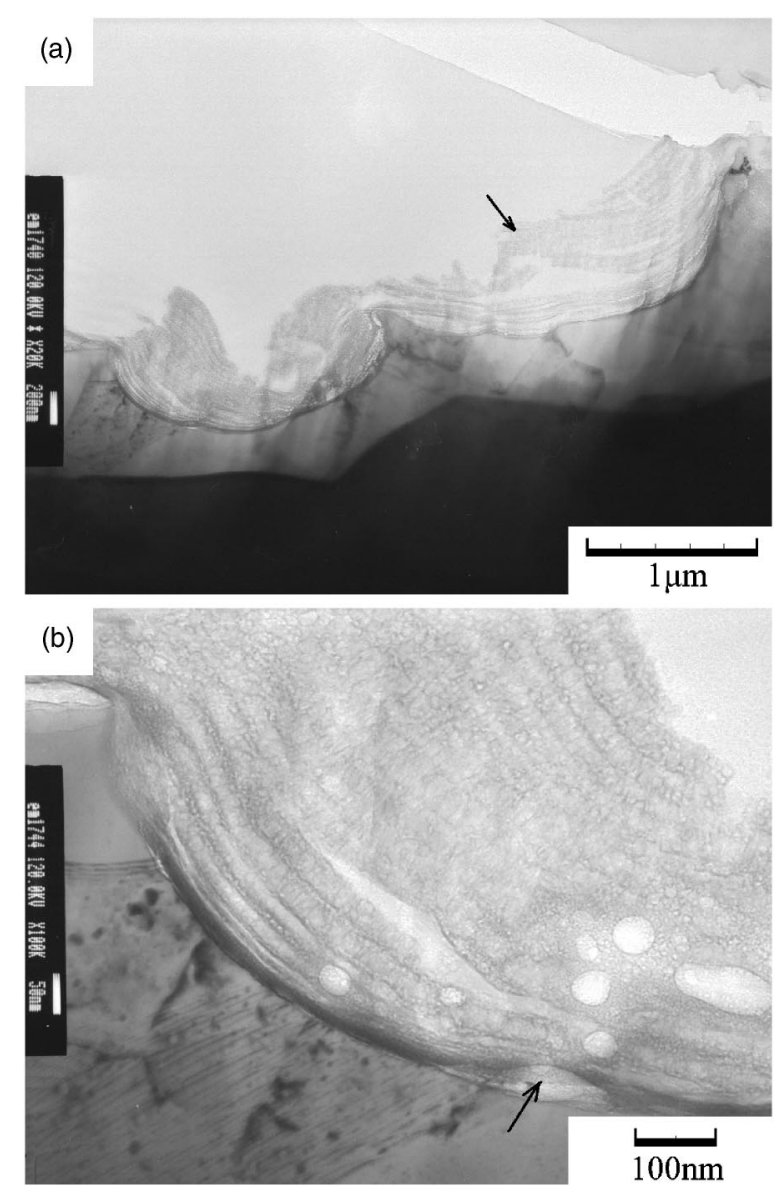

Figure 4. Cross-sectional TEM micrographs of the plate etched at $t_{\mathrm{a}} / t_{\mathrm{c}}=2$ : (a) the overall structure of the etch film and (b) a close-up view showing the morphology of the layered etch film.

substrate, as evident from the microvoids (the arrow in Fig. 4b) residing at the interface. Several round microvoids were also observed within the etch film.

Figure 5a shows that the layered etch films were still observed as $t_{\mathrm{a}} / t_{\mathrm{c}}$ was increased to 5 . The plate surface was also intimately covered with a dark-contrast layer under which microvoids were observed, as marked by the arrow in Fig. 5b. These voids had a diameter ranging from 10 to $20 \mathrm{~nm}$, which was close to the spacing between two dark-contrast layers. Figure 6 shows that the aluminum surface was locally dotted with relatively deep and large pits when $t_{\mathrm{a}} / t_{\mathrm{c}}$ exceeded 10. Inside the pit, the layered etch film was no longer observed. Instead, the pit was filled with nonlaminar etch products (Fig. 6a and b). Figure 6d and e show the EDS spectra from the areas marked as A and B shown in Fig. 6b. From these spectra, the dark-contrast (marked as A) and gray-contrast (marked as B) areas were characterized as the etch product and the M-Bond epoxy, respectively. The former was amorphous as evident from the diffused halos shown in the SAD pattern (Fig. 6c).

Figure 7a summarizes the thickness of the dark-contrast layer as a function of $t_{\mathrm{a}} / t_{\mathrm{c}}$ ratio. The thickness notably decreased with increasing $t_{\mathrm{a}} / t_{\mathrm{c}}$. This observation indicates that the thickness of the dark-contrast layers decreases as the charge passed per anodic halfcycle increases. Figure $7 b$ shows that the average size of the individual hemispherical pit increased with increasing $t_{\mathrm{a}} / t_{\mathrm{c}}$, indicating that the pit grew via dissolution of aluminum during the anodic half-cycle.

AA1050 plates etched at $t_{c} / t_{a}$ exceeding $1 .-$ Figure 8-10 summarize the effects of increasing the charge imposed during each cathodic half-cycle. Figure 8 shows an example of the cross section
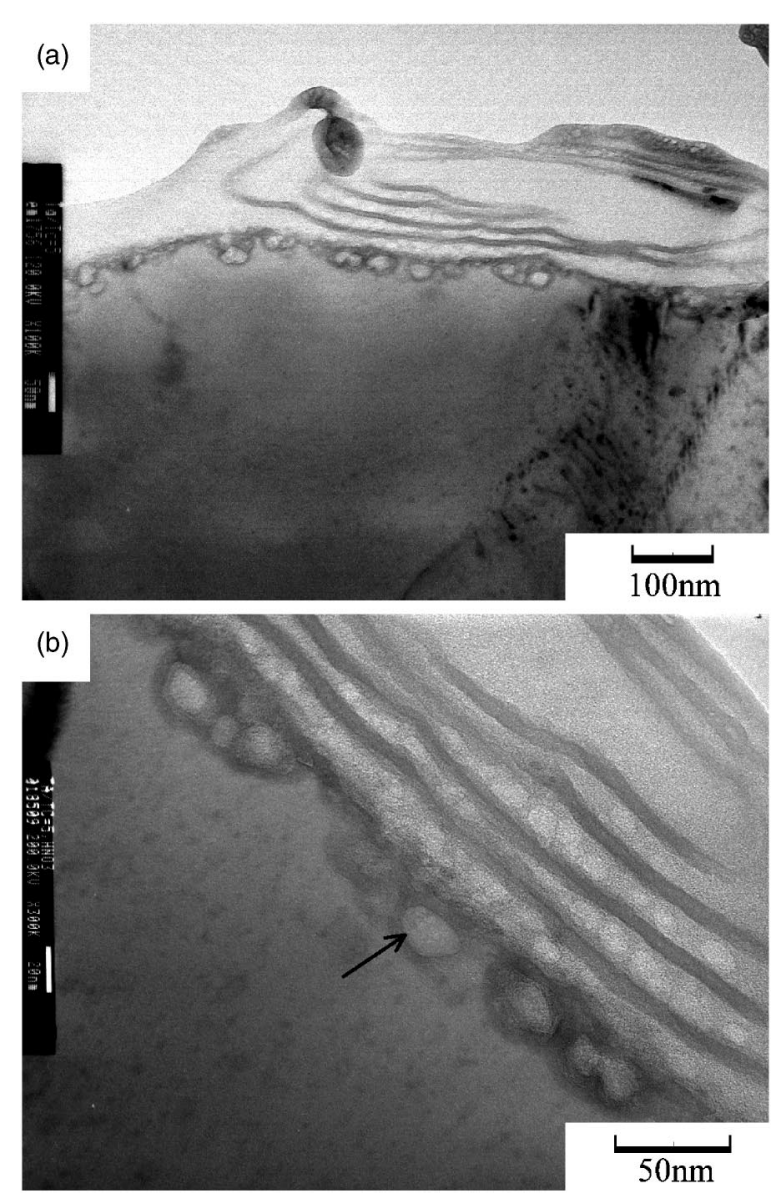

Figure 5. Cross-sectional TEM micrographs of the plate etched at $t_{\mathrm{a}} / t_{\mathrm{c}}=5$ : (a) the overall structure of the etch film and (b) a close-up view showing microvoids at the interface.

of the plate etched at $t_{\mathrm{c}} / t_{\mathrm{a}}=5$. This micrograph shows that the individual pits were entirely filled with etch films. The etch films typically consisted of three layers with a relatively thin dark-contrast layer overlaying the substrate, a light-contrast layer as an intermediate layer, and a relatively thick dark-contrast layer as the major overlay. EDS analyses show that the two dark-contrast layers had similar composition. Both contained the signals of aluminum and oxygen, while the light-contrast layer was primarily the M-Bond epoxy. The same etch film structure was observed when $t_{\mathrm{c}} / t_{\mathrm{a}}$ was further increased to 10 , when the thickness of the relatively thick dark-contrast layer had a thickness close to the depth of the pit, as shown in Fig. 9.

Since the size of the pits decreased with increasing $t_{\mathrm{c}} / t_{\mathrm{a}}$ as evident by comparing Fig. 3 to Fig. 8 and 9, the pits were scarcely observed when $t_{\mathrm{c}} / t_{\mathrm{a}}$ was increased to 20 . Nevertheless, a thick etch film was observed on the surface of the etched plate (Fig. 10b). In this figure parts of the etch film had already peeled off the aluminum surface, as illustrated by the light-contrast layers at the interface between the dark-contrast layer and the substrate (the arrow in Fig. 10b). Furthermore, the light-contrast areas at the interface were hemispherical and convex toward the free surface, suggesting the etch film was pushed out of the substrate by certain forces exerted at the interface. In addition to the thick single-layered etch film, Fig. 10c shows that some etch films contained two dark-contrast layers, which were separated by a light-contrast layer that was mainly M-Bond epoxy. Figure 10a shows that the etch film was thick enough to mask the real topography of the plate. Furthermore, the etch film contained cracks formed presumably due to desorption of 

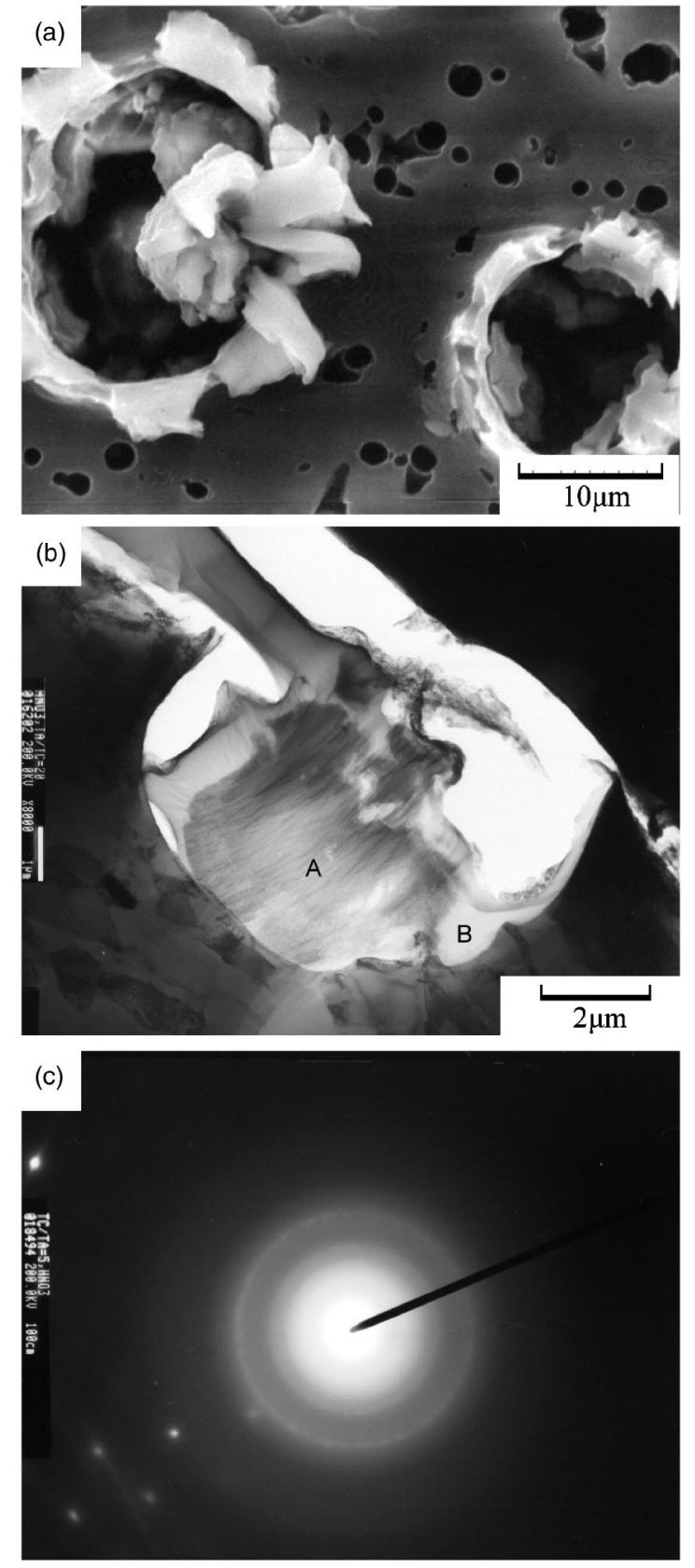

(d)

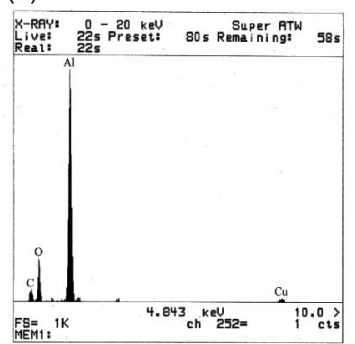

(e)

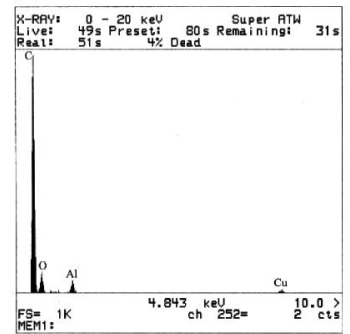

Figure 6. (a) and (b) are, respectively, the surface morphology and crosssectional TEM micrograph of the plate etched at $t_{\mathrm{a}} / t_{\mathrm{c}}=20$; (c) selected area electron diffraction pattern from the area marked as A in (b); (d) and (e) EDS spectra taken from the areas marked as A and B in (b), respectively.

water molecules when the sample was dried in the atmosphere overnight. Finally, higher $t_{\mathrm{c}} / t_{\mathrm{a}}$ resulted in a thicker dark-contrast layer and a smaller individual pit (Fig. 7a and b).
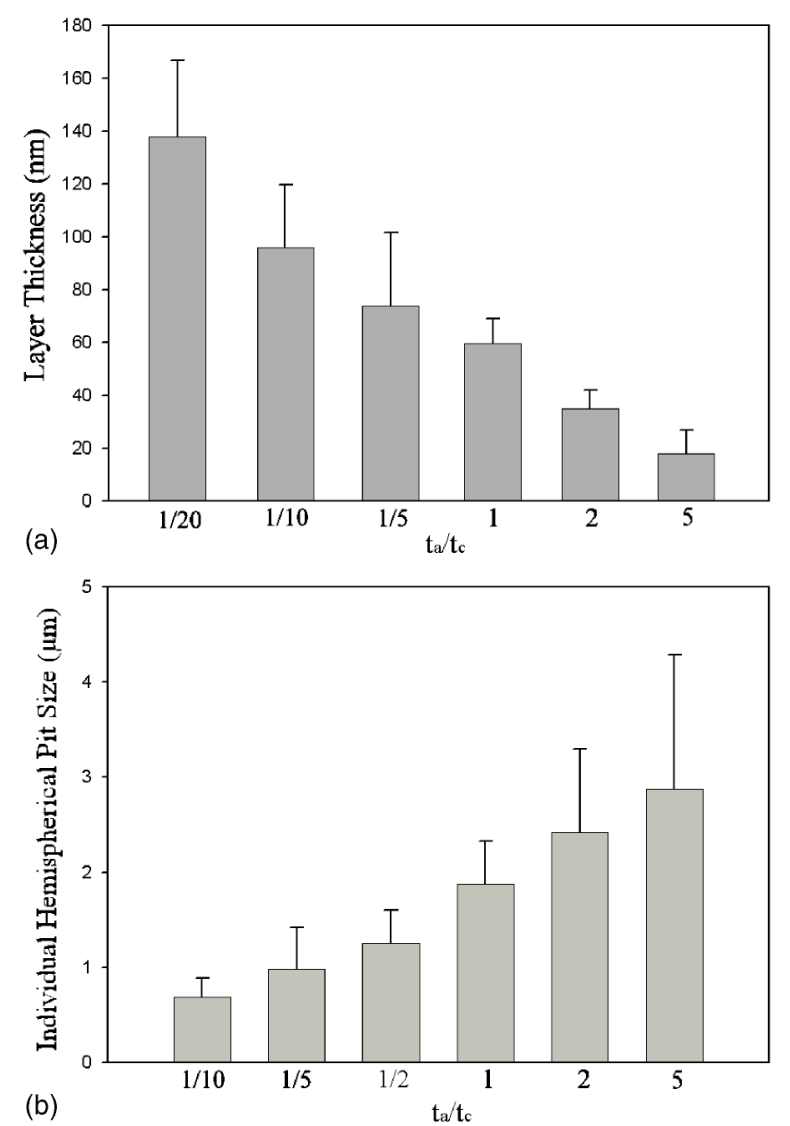

Figure 7. Thickness of the dark-contrast layer and average size of the individual hemispherical pit as a function of the ratio of positive to negative charges passed during each anodic and cathodic half-cycle.

\section{Discussion}

When etched in hydrochloric or nitric acids using alternating currents, it has been shown that the aluminum plates are covered with etch products that markedly mask the real surface topography. ${ }^{5-8,11-15,22,23}$ For etching in hydrochloric acid, it is generally accepted that the etch film forms during the cathodic half-cycle due to the high interfacial $\mathrm{pH}$ induced by reduction of protons. ${ }^{7,10,12,14,22}$ According to this mechanism, hydrogen evolution during the cathodic half-cycle results in the increase of interfacial $\mathrm{pH} . \mathrm{Al}^{3+}$ ions, created during the anodic half-cycle, are subsequently

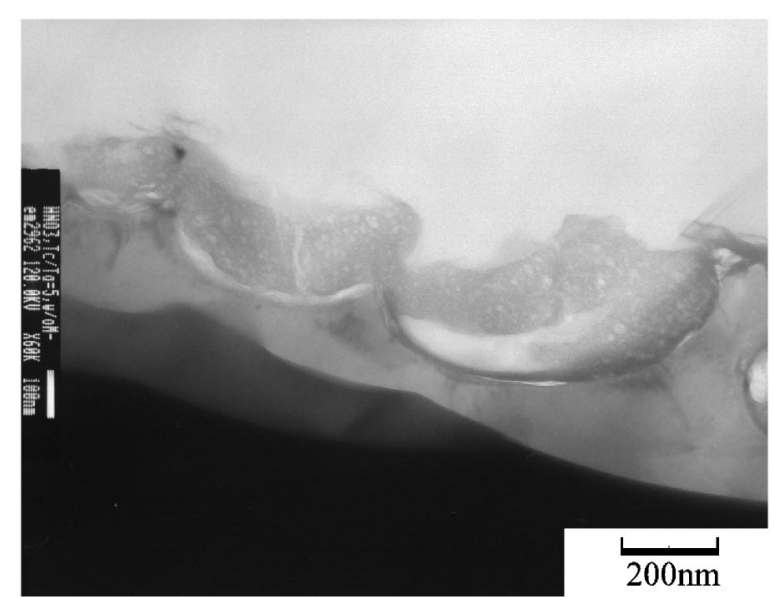

Figure 8. Cross-sectional TEM micrograph of the plate etched at $t_{\mathrm{c}} / t_{\mathrm{a}}=5$. 


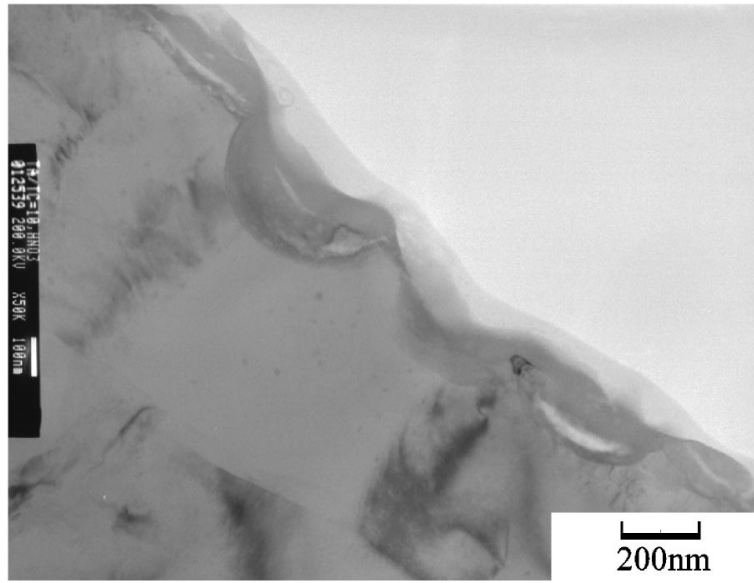

Figure 9. Cross-sectional TEM micrograph of the plate etched at $t_{\mathrm{c}} / t_{\mathrm{a}}$ $=10$.

deposited as amorphous aluminum hydroxides when the $\mathrm{pH}$ exceeds 5. The feasibility of the aforementioned mechanism has been proved by Dyer and Alwitt. ${ }^{7}$ As for etching in nitric acid-based solution, the etch film shows a layered structure. ${ }^{13,15,23}$ The layered film has been considered as a characteristic of porous anodic films. ${ }^{13}$ However, further explanations about the formation of the layered etch film have not been reported yet.

The present study also found that the layered etch film forms via the stacking of dark-contrast and light-contrast layers. These darkcontrast layers are presumably amorphous aluminum hydroxides, while the light-contrast layer is just the gap between two successive dark-contrast layers. Furthermore, the thickness of the hydroxide layer increases with increasing the charges imposed during the cathodic half-cycle (Fig. 7a). This result signifies that the etch film forms predominantly during the cathodic half-cycle when local $\mathrm{pH}$ increases due to hydrogen discharge. This is in good consistence with the mechanisms for the formation of the etch films on aluminum etched in hydrochloric acid. ${ }^{7,12,14}$

Because the hydroxide layers copy the shape of the pit, the same surface location of the aluminum substrate must be undergoing dissolution-passivation during successive cycles for the layers of the etch film to regularly stack and assume exactly the shape of the pit. The pit, if made passive by the etch film, must be depassivated during the following cycles so that the attack can continue on the existing pit. Figure 11 shows a schematic representation illustrating dissolution-passivation of the pits as etching proceeds. It is well known that a thin oxide layer forms once aluminum is exposed to the atmosphere. ${ }^{24}$ After alkaline cleaning, the aluminum substrate can be covered with a thin oxide or hydroxide layer. This layer is simply indicated as the oxide layer in Fig. 11a. Upon immersing in nitric acid, the substrate is locally attacked during the anodic halfcycle, creating the etch pits whose bases contain significant amounts of $\mathrm{Al}^{3+}$ ions during the end of the anodic half-cycle (Fig. 11b). During the subsequent cathodic half-cycle, protons inside the etch pit are reduced, creating a high $\mathrm{pH}$ environment for the deposition of aluminum hydroxide, ${ }^{25}$ which shows up as the dark-contrast layer in TEM (Fig. 11c). Once the wall of the etch pit is covered with aluminum hydroxide, it can be passivated. However, this hydroxide layer can also be damaged by the hydrogen bubbles resulting from proton reduction, as illustrated by the presence of microvoids inside the hydroxide layer and on the surface of the aluminum substrate. Like the mechanical stress resulting from the gas pressure exerted at the oxide-aluminum interface causes the formation of blister on aluminum in chloride solutions, ${ }^{1,20,21}$ the pressure accumulated within the hydrogen bubbles or produced as the escape of the bubbles creates stresses that might induce additional defects in the hydroxide layer. At the beginning of the subsequent anodic half-
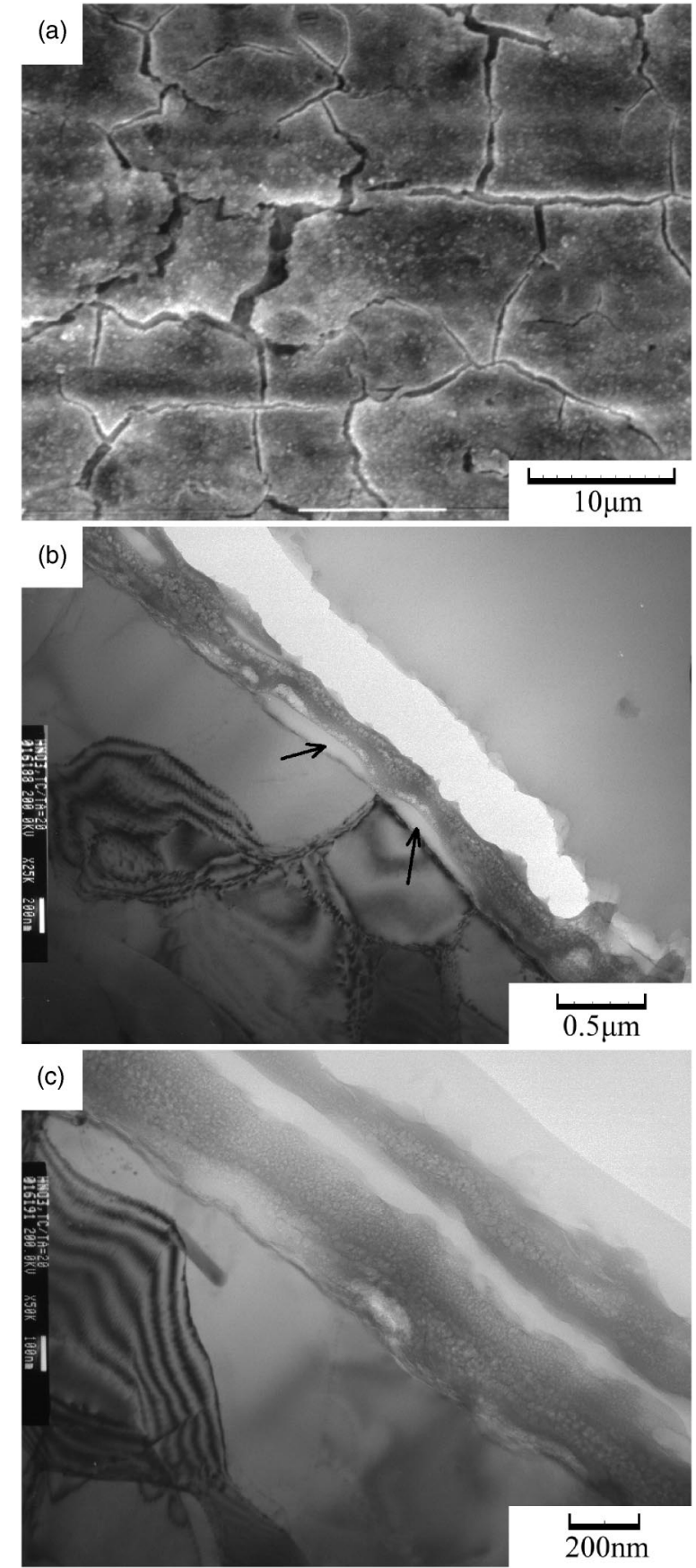

Figure 10. (a) Surface morphology of the plated etched at $t_{\mathrm{c}} / t_{\mathrm{a}}=20$; (b) and (c) are the cross-sectional TEM micrographs.

cycle, new pits will form at discrete sites along the wall of the existing pit, preferably at those dotted with microvoids or defects, or at the original aluminum surface covered with the oxide layer (Fig. 11d). As the anodic half-cycle continues, the dissolution of aluminum at these sites can cause separation of the hydroxide layer from the substrate (Fig. 11e). This leads to the exposure of bare substrate to further attack as the anodic half-cycle proceeds. The formation and subsequent detachment of the hydroxide layer proceeds alternately with continued etching (Fig. 11f and g). As a result, dissolution and passivation of the same pit results in the layered etch film observed in the present study. Dissolution-passivation will continue on the same pit until the layered etch film becomes very thick when the conduction resistance of the etch film is large enough to stifle pit growth. Then, the pits, if formed, must be nucleated elsewhere, lead- 


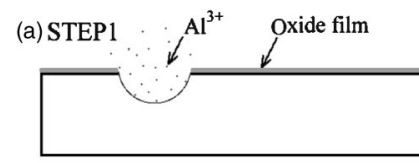

(b) STEP2

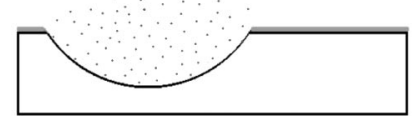

(c) STEP3

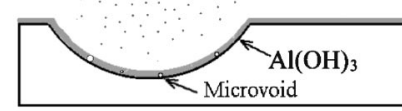

(d) STEP4
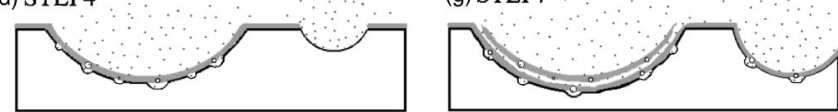

Figure 11. Schematic representations illustrating the formation of the layered etch film.

ing to the formation of the overall convoluted pitted structure. The existence of an aluminum hydroxide layer intimately contacting the substrate can be associated with the thick layered etch film completely filling in the mouth of the pit, because the thick etch film makes electrolyte diffusion difficult and imposes a high conduction resistance.

\section{Conclusions}

The present study details the microstructure of the etch film on AA1050 aluminum plates etched in nitric acid using the square alternating current. The layered etch film contained alternate darkcontrast and light-contrast layers. The dark-contrast layer consisted of aluminum hydroxide. Since the hydroxide layer always copied the shape of the pit, it appears to form directly on the surface of the substrate. In contrast, the light-contrast layer was presumably the gap between two successive hydroxide layers.

As the charge passed during each cathodic half-cycle increased, the thickness of the hydroxide layer increased, while the average size of the pits decreased. The formation of aluminum hydroxide was apparently related to hydrogen discharge during the cathodic half-cycle, whereas the pits formed during the anodic half-cycle. The existing pit was probably passivated once it was fully covered with a layer of aluminum hydroxide. However, voids at the hydroxide-substrate interface and the resulting partial detachment of the hydroxide layer adjacent to the substrate were frequently observed. Hydrogen bubbles building up during the cathodic halfcycles contributed to the local breakdown of the hydroxide layer, which in turn enhanced pit nucleation and aluminum dissolution at discrete sites on the pit wall at the beginning of the subsequent anodic half-cycle. This dissolution caused separation of the hydroxide layer from the substrate and exposed the bare aluminum to general attack as the anodic half-cycle continued. During the subsequent cathodic half-cycle, the pit was again covered with a hydroxide layer. The layered etch film apparently formed via dissolutionpassivation on the same pit.

\section{Acknowledgments}

This research was supported by the National Science Council of Taiwan under grant no. 892216E212003. The authors thank S. H. Hsieh, China Steel Corporation, for his invaluable discussion, and L. C. Wang, National Sun Yat-sen University, for her assistance with the TEM work. This study made use of the Electron Microscopes of National Sun Yat-sen University and National Chung-hsing University, supported by the National Science Council, Taiwan.

National Taiwan University assisted in meeting the publication costs of this article.

\section{References}

1. E McCafferty, Corros Sci, 45, 1421 (2003).

2. Z. Szklarska-Smialowska, Corros. Sci., 41, 1743 (1999).

3. R. T. Foley, Corrosion (Houston), 42, 277 (1986).

4. C. M. Liao and R. P. Wei, Electrochim. Acta, 45, 881 (1999).

5. G. E. Thompson and G. C. Wood, Corros. Sci., 18, 721 (1978).

6. T. Suzuki and Y. Hayashi, Kinzoku Hyomen Gijutsu, 30, 541 (1979).

7. C. K. Dyer and R. S. Alwitt, J. Electrochem. Soc., 128, 300 (1981).

8. K. V. Quang, F. Brindel, G. Laslaz, and R. Buttoudin, J. Electrochem. Soc., 130, 1248 (1983).

9. R. S. Alwitt, H. Uchi, T. R. Beck, and R. C. Alkire, J. Electrochem. Soc., 131, 13 (1984).

10. W. M. Moore, C. T. Chen, and G. A. Shirn, NACE Corrosion, 40, 644 (1984)

11. J.-C. Huang, Proc. Symp. Aluminum Surface Treatment Technology, R. S. Alwitt, Editor, The Electrochemical Society, Pennington, NJ (1986).

12. P. Laevers, H. Terryn, J. Vereecken, and G. E. Thompson, Corros. Sci., 35, 231 (1993).

13. M. P. Amor and J. Ball, Corros. Sci., 40, 2171 (1998).

14. H. Terryn, B. Kernig, A. Hubin, and P. Laevers, Trans. IMF, 78, 29 (2000).

15. C. S. Lin, C. C. Chang, and S. H. Hsieh, J. Electrochem. Soc., 147, 3647 (2000).

16. Z. A. Foroulis and M. J. Thubrikar, J. Electrochem. Soc., 122, 1296 (1975).

17. H. Böhin and H. H. Uhlig, J. Electrochem. Soc., 116, 906 (1969).

18. C. F. Lin, M. D. Porter, and K. R. Hebert, J. Electrochem. Soc., 141, 96 (1994).

19. C. F. Lin and K. R. Hebert, J. Electrochem. Soc., 137, 3723 (1990).

20. C. B. Bargeron and R. B. Givens, Corrosion (Houston), 36, 618 (1980).

21. C. B. Bargeron and R. B. Givens, J. Electrochem. Soc., 124, 1845 (1977).

22. P. Laevers, H. Terryn, and J. Verrecken, Trans. IMF., 70, 105 (1992).

23. C. S. Lin and S. M. Fu, J. Electrochem. Soc., 148, C240 (2001).

24. M. S. Hunter and P. Fowle, J. Electrochem. Soc., 103, 482 (1956)

25. M. Pourbaix, Atlas of Electrochemical Equilibria in Aqueous Solutions, 2nd ed., NACE, Houston, TX (1974). 